\title{
Stark schwankender Blutdruck spricht für geistigen Abbau
}

- Schwanken die Blutdruckwerte älterer Patienten stark von Arztbesuch zu Arztbesuch, deutet dies auf kognitive Einschränkungen hin.

In einer Studie mit 201 Senioren im Alter über 70 hatten japanische Hypertensiologen deren Blutdruckwerte ein Jahr lang monatlich gemessen. Alle Probanden wur- den zudem mittels Mini-Mental-State-Test (MMST) auf ihre kognitive Leistungsfähigkeit untersucht und anhand der ReisbergSkala eingestuft (1-7, Werte von 3-7 gelten als leichte bis sehr schwere Demenz). Bei der Auswertung ergaben sich signifikante Zusammenhänge zwischen der Differenz des maximal und minimal gemes-

\section{Glosse}

\section{Akademisches Gelächter}

- Haben Sie heute schon gelacht? Wenn ja: Sind Sie überhaupt zum Lachen qualifiziert? Haben Sie einen Abschluss in Gelotologie? Den Begriff haben Sie sicher schon gehört. Das ist die Lachforschung. Mit einem Bachelor in Gelotologie dürfen Sie leise in sich hinein kichern, für wieherndes Gelächter brauchen Sie den Master.

Uns Deutschen sagt man ja immer noch nach, dass wir keinen Humor hätten und allenfalls zu sardonischem Gelächter fähig seien. Um herauszufinden, was Gelotologen so treiben, müssen wir uns daher an kompetentere ausländische Quellen halten. In der Schweiz wurden wir fündig. Neues "Aus der Welt der Lachforschung" verspricht www.humor.ch. Da lesen wir z. B.: "Automaten: schmerzempfindliche Gartenfrüchte". Ha ha ... aha ... hm. Die Gelotologie ist eine noch junge Wissenschaft, erfahren wir da und „Humor ist eine ernste Sache". Das hatten wir befürchtet.

Andererseits: Bei ernsten Sachen können wir doch auch mithalten. Und siehe da: In Leipzig gibt es tatsächlich ein „Deutsches Institut für Humor (DIH) ${ }^{\circledR}$ (www.humorinstitut.de). Die Chefin, Eva Ullmann, lächelt uns auf der Homepage nett entgegen. Das strahlt schon eine gewisse Kompetenz in Sachen Gelotologie aus, obwohl Frau Ullmann „nur" gelernte Pädagogin ist.
Das DIH bietet Humortraining an. Die Kurse haben Titel wie: „Humor als Ressource im Arbeitsalltag". Humor und Arbeitsalltag? Wie geht das zusammen? Schließt das eine nicht das andere aus, außer vielleicht bei Berufskomikern? Ressourcen werden ausgebeutet. Sollen humorvolle Arbeitnehmer also besonders ausgebeutet werden? Bekommen die dann wenigstens einen Bonus? Darf man mit einer Belobigung rechnen, wenn man auf Anordnungen seines Vorgesetzten mit schallendem Gelächter reagiert?

Diese Fragen zeigen, dass der Schreiber dieser Zeilen vom Lachen rein gar nichts versteht. Kein Wunder: Erstens ist er Deutscher und zweitens hat er nie Gelotologie studiert. Mangels Qualifikation wird er also künftig auf Humor und Lachen verzichten müssen, denn: „Humor ist nicht immer die beste Medizin“, warnt das DIH. „Es kann also sein, dass nicht alle Komponenten von Humor gut für die Gesundheit und das Wohlbefinden sind." Der Ausruf "Ich lach mich krank" erscheint so in völlig neuem Licht.

Empfehlenswert ist Lachen hingegen bei schwerer COPD. Lächeln und leichtes Lachen reduziert nämlich das Lungenvolumen und lindert so die COPD-Symptome. „Schenke COPD-Patienten ein Lächeln!" rät daher das DIH.

Womit sich die Frage stellt: Lächelt Frau Ullmann wirklich nur nett, oder hat sie einen Raucherhusten?

REINHOLD MANHART . senen systolischen sowie diastolischen Blutdrucks und den kognitiven Funktionen - je schlechter diese, desto größer jene. Auch der prozentuale Anteil, den die mittlere Schwankung des systolischen Blutdrucks (Standardabweichung) am systolischen Mittelwert aller zwölf Messungen hatte, korrelierte mit den geistigen Leistungen: je größer der Anteil, desto niedriger der MMST-Wert und desto höher die Bewertung mit der Reisberg-Skala. Nicht nur die Höhe des Blutdrucks, sondern auch seine Schwankungen korrelieren demnach mit kognitiven Beeinträchtigungen.

$\mathrm{RB}$.

J Hypertens 2012, online 21. Juni; doi: 10.1097/ HJH.0b013e3283552735

\section{ENTWARNUNG}

\section{Keine Nickelallergie durch Zahnspange}

- Immer mehr Menschen, insbesondere Jugendliche, leiden an einer Nickelallergie. $\mathrm{Ob}$ eine solche durch eine Zahnspange ausgelöst werden kann, wurde in einer Studie an jungen Mädchen mit einer fixen Zahnschiene untersucht. Dabei ergab sich nach einem Jahr kein erhöhtes Risiko für eine Nickelsensibilisierung. Von den 30 Jugendlichen mit Zahnspange zeigten zwar 7 ein positives Ergebnis auf eine Epikutantestung, jedoch oh ne klinische Symptome

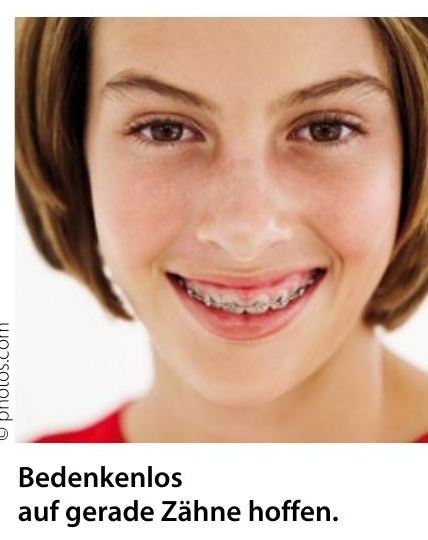
im Bereich der Mundschleimhaut. „Die Angst vor einer Nickelallergie ist unbegründet; wenn eine Zahnspange nötig ist, sollte sie eingesetzt werden", so die Empfehlung der Experten.

STI =

Update-Seminar Allgemeinmedizin, München, 28.-29.4.2012 